\title{
ASYMMETRY IN THE LATTICE OF KERNEL FUNCTORS
}

\author{
by ANA M. DE VIOLA-PRIOLI and JORGE E. VIOLA-PRIOLI
}

(Received 10 October, 1989)

Much of the research done by different authors on the lattice of kernel functors (equivalently, linear topologies) has been summarized by Golan in [2]. More recently, the rings whose lattices of kernel functors are linearly ordered were introduced in [3] as a categorical generalization of valuation rings in the non-commutative case. Results (and examples) in [3] show that there is an abundance of non-commutative rings $R$ whose lattices $\mathbb{K}(R)$, both in Mod- $R$ and $R$-Mod, are simultaneously linearly ordered; however, the question of the symmetry of this condition remained open. Here we will prove that, for every natural number $n \geq 3$, there exists a ring $R_{n}$ such that $\mathbb{K}\left(\operatorname{Mod}-R_{n}\right)$ is a linearly ordered lattice of $n$ elements, whereas $\mathbb{K}\left(R_{n}-\mathrm{Mod}\right)$ is not linearly ordered.

Throughout, familiarity with [3] is assumed but, instead of $\mathbb{K}(R)$ we are denoting by $\mathbb{K}(\operatorname{Mod}-R)$ (respectively $\mathbb{K}(R-\mathrm{Mod})$ ) the lattice of kernel functors on Mod- $R$ (respectively $R$-Mod), to specify sides.

We begin by considering the case $n=3$.

Let $K$ be a field, $f: K \rightarrow K$ a field monomorphism which is not onto and let $L$ denote $f(K)$. Consider the ring $S$ of twisted power series, that is, $S=\left\{\sum x^{j} a_{j} ; a_{j} \in K\right\}=K[[x ; f]]$ with the usual addition and $a x=x f(a)$, for every $a$ in $K$.

The only right ideals of $S$ are $S \supset x S \supset x^{2} S \supset \ldots$, and thus $\mathbb{K}(\operatorname{Mod}-S)$ is linearly ordered by [3, Lemma 7]. Let $R$ be the ring $S / x^{2} S$. Therefore $R=\{a+x b ; a, b \in K\}$, where $k x=x f(k)$ and $x^{2}=0$. Clearly $R$ has only three right ideals, $R, x R$, and (0), and $\mathbb{K}(\operatorname{Mod}-R)$ is linearly ordered.

It is obvious that for every $L$-subspace $V$ of $K$ the set $x V$ is a left ideal of $R$. Moreover, these are the only proper left ideals of $R$. In fact, given ${ }_{R} I \subsetneq R$ and $a+x b \in I$, it follows that $a=0$ (otherwise an inverse can be found since $f$ is a monomorphism) and therefore $I \subseteq x K$. Let $V$ denote $\{u \in K ; x u \in I\}$. This set is an $L$-subspace of $K$ since given $u \in K$ and $t \in L, t=f(k)$ for a certain $k \in K$; so $x(t u)=x f(k) u=k(x u) \in k I \subseteq I$.

Once we have obtained the left ideals of $R$ we are in a position to prove the following lemma.

LEMMA. For the ring $R$ as above, the following conditions are equivalent:

(a) $\mathbb{K}(R$-Mod) is linearly ordered;

(b) for any pair $V, W$ of $L$-subspaces of $K$, there exists a finite set $\left\{x_{1}, \ldots, x_{s}\right\}$ of non-zero elements of $K$ such that either $V x_{1} \cap \ldots \cap V x_{s} \subseteq W$ or $W x_{1} \cap \ldots \cap W x_{s} \subseteq V$.

Proof. (a) $\Rightarrow$ (b). We may assume $V$ and $W$ are not comparable, since otherwise $x_{1}=1$ will do. Set $I=x V$ and $J=x W$. By [3, Proposition 1], there exists in $R$ a finite set $\left\{r_{i}\right\}_{1}^{p}=\left\{a_{i}+x b_{i}\right\}_{1}^{p}$ such that $I \supseteq\left(J:\left\{r_{i}\right\}_{1}^{p}\right)$, for instance. If $a_{i}=0$ for every $i$ then $x K r_{i}=x K x b_{i} \in x^{2} R=(0)$ and $x K \subseteq\left(J:\left\{r_{i}\right\}_{1}^{p}\right) \subseteq I=x V$, which implies $V=K \supseteq W$, a contradiction. So one may assume that $a_{1}, \ldots, a_{s}$ are non-zero $(s \leq p)$ and $a_{s+1}=\ldots=a_{p}=$ 0 , and proceed to prove that $W a_{1}^{-1} \cap \ldots \cap W a_{s}^{-1} \subseteq V$. In fact, $k \in \bigcap_{j=1}^{s} W a_{j}^{-1}$ implies 
$k=w_{j} a_{j}^{-1}$ for some $w_{j} \in W$, and therefore

$$
x k r_{i}= \begin{cases}x w_{i} a_{i}^{-1} a_{i} \in x W=J & \text { if } i \leq s \\ x k x b_{i}=0 \in J & \text { if } s<i\end{cases}
$$

Hence $x k \in\left(J:\left\{r_{i}\right\}_{1}^{p}\right) \subseteq I=x V$ and so $k \in V$.

(b) $\Rightarrow$ (a). We are given two left $R$-ideals $I$ and $J$, which may be taken of the form $x V, x W$, respectively, for certain subspaces $V$ and $W$. By hypothesis, there exist non-zero elements $k_{1}, \ldots, k_{s}$ in $K$ such that $W k_{1} \cap \ldots \cap W k_{s} \subseteq V$, for instance. Set $C=$ $\left\{k_{1}^{-1}, \ldots, k_{s}^{-1}\right\}$ and let us check that $I \supseteq\left(J:{ }_{R} C\right)$. In fact, if $a+x b \in(J: C)$ then $a k_{i}^{-1}+x b k_{i}^{-1}=(a+x b) k_{i}^{-1} \in x W$ for every $i$, and so $a=0$ and $b k_{i}^{-1} \in W$ for every $i$. Since $b \in \bigcap W k_{i} \subseteq V$, it follows that $a+x b=x b \in x V=I$. Now [3, Proposition 1] ensures that $\mathbb{K}(R-\mathrm{Mod})$ is linearly ordered.

Observe that we will be done if a particular choice of $K$ and $f$ enables us to violate condition (b).

In the rational function field in infinitely many indeterminates $K=$ $\mathbb{Q}\left(x_{1}, \ldots, x_{n}, \ldots\right)$, take the ring monomorphism $f: K \rightarrow K$ given by $f\left(x_{j}\right)=x_{j+1}$. Therefore $f(K)=\mathbb{Q}\left(x_{2}, x_{3}, \ldots\right)=L$ and $K=L\left(x_{1}\right)=L(t)$ if we set $x_{1}=t$. Consider the $L$-subspaces $V=L[t]$ and $W=t^{-1} L\left[t^{-1}\right]$, and notice that, given arbitrary non-zero elements $f_{1} / g_{1}, \ldots, f_{s} / g_{s}$ of $K$, the polynomial $f=\prod_{1}^{s} f_{i} \notin W$. However $f=\left(\prod_{i \neq j} f_{i}\right) g_{j} f_{j} / g_{j} \epsilon$ $V f_{j} / g_{j}$ for every $j$, which implies that $\bigcap_{1}^{s} V f_{j} / g_{j} \nsubseteq W$. On the other hand, pick an integer $m>\sum_{1}^{s} \operatorname{deg}\left(f_{i}\right)+\operatorname{deg}\left(g_{i}\right)$ to obtain the element $f / t^{m} \notin V$. Moreover

$$
f / t^{m}=\frac{\left(\prod_{i \neq j} f_{i}\right) g_{j}}{t^{m}} f_{j} / g_{j} \in W f_{j} / g_{j}
$$

for every $j$, and therefore $\bigcap_{1}^{s} W f_{j} / g_{j} \nsubseteq V$.

So far we have constructed a ring $R$ such that $\mathbb{K}(\operatorname{Mod}-R)$ is a linearly ordered lattice of three elements, whereas $\mathbb{K}(R$-Mod $)$ is not linearly ordered.

Let us denote by $R_{3}$ the ring just constructed and proceed to tackle the case of arbitrary $n$. Consider $S$ as before and define $R_{n}=S / x^{n-1} S$; this is a right chain ring and $\mathbb{K}\left(\operatorname{Mod}-R_{n}\right)$ is a linearly ordered lattice of $n$ elements, by [3, Corollary 7]. However, if we choose $K$ and $f$ as above, $\mathbb{K}\left(R_{n}\right.$-Mod) linearly ordered would force $\mathbb{K}\left(R_{3}\right.$-Mod) to be linearly ordered, as $R_{3}$ is an epimorphic image of $R_{n}$, and [3, Proposition 2] would apply.

COMmENTs. Generalizations to the non-artinian case would be obtained by considering non-finite ordinal numbers $\tau$ and a ring of type $\tau$ as in [1, p. 312]. The argument still applies since $R_{3}$ is an epimorphic image of such a ring. Details are omitted. Finally, the case $n=2$ has to be ruled out since $K(\operatorname{Mod}-R)=\{\mathbb{Q}, \mathbb{O O}\}$ if and only if $R$ is a simple artinian ring, if and only if $\mathbb{K}(R-\mathrm{Mod})=\{\mathbb{0}, 00\}$. 


\section{REFERENCES}

1. P. M. Cohn, Free rings and their relations (Academic Press, 1971).

2. J. S. Golan, Linear topologies on a ring: an overview, Research Notes in Mathematics No. 159 (Pitman, 1987).

3. A. M. de Viola-Prioli and J. Viola-Prioli, Rings whose kernel functors are linearly ordered, Pacific J. Math. 132 (1988), 21-34.

Universidad Simón Bolívar

Caracas, Venezuela 